\title{
Cardiovascular risk factors and $\gamma$-glutamyltransferase fractions in healthy individuals
}

\author{
Maria Franzini ${ }^{1, a, *}$, Aldo Paolicchi ${ }^{2, a}$, Irene \\ Fornaciari $^{1}$, Virginia Ottaviano ${ }^{2}$, Vanna Fierabracci ${ }^{2}$, \\ Maristella Maltinti ${ }^{3}$, Andrea Ripoli ${ }^{3}$, Luc $\mathbf{Z y w}^{3}$, \\ Fabrizio Scatena ${ }^{4}$, Claudio Passino ${ }^{1,3}$, Alfonso \\ Pompella ${ }^{2}$ and Michele Emdin ${ }^{3, a}$ \\ ${ }^{1}$ Scuola Superiore S. Anna, Pisa, Italy \\ ${ }^{2}$ Department of Experimental Pathology, University of \\ Pisa, Italy \\ ${ }^{3}$ Division of Cardiovascular Medicine and Clinical \\ Chemistry, G. Monasterio Foundation, CNR-Regione \\ Toscana, Pisa, Italy \\ ${ }^{4}$ UO Immunoematologia 2, Azienda Ospedaliera \\ Universitaria Pisana, Pisa, Italy
}

\begin{abstract}
Background: Serum $\gamma$-glutamyltransferase activity (GGT), even when within its normal reference range, catalyzes low density lipoprotein oxidation in vitro and predicts cardiovascular events. Of the four GGT fractions (b-GGT, m-GGT, sGGT, and f-GGT) recently identified in blood, only b-GGT is found within atherosclerotic plaques. Our goal was to identify the determinants of the GGT fractions (b-, m-, s-, and f-GGT) and their association with established cardiovascular risk factors in healthy subjects.

Methods: Multiple linear regression analysis was applied to estimate the association of fractional GGT with gender, age, body mass index, arterial pressure (AP), plasma glucose, alanine aminotransferase (ALT), high and low density lipoproteins (LDL-C) cholesterol (HDL-C), triglycerides (TG) and C-reactive protein (CRP) in 200 healthy subjects.

Results: All GGT fractions were associated with ALT; bGGT with AP, TG, and CRP; m-GGT with LDL-C, TG and CRP; s-GGT with TG and CRP, and f-GGT only with LDL$\mathrm{C}$, whereas gender was associated with s-GGT and f-GGT only.

Conclusions: In healthy individuals, cardiovascular risk factors are associated with high molecular weight GGT fractions, namely with b-GGT, the only form present within the plaque. This finding adds to the present knowledge concerning the relevance of GGT, within its reference range, for atherosclerosis-related events.
\end{abstract}

Clin Chem Lab Med 2010;48:713-7.

\footnotetext{
${ }^{a}$ MF, AP, ME equally contributed to the study.

*Corresponding author: Maria Franzini, Scuola Superiore

Sant'Anna, c/o G. Monasterio Foundation, CNR-Regione Toscana,

Via Giuseppe Moruzzi 1, 56124 Pisa, Italy

Phone: +(39)-050-3153309, Fax: +(39)-050-3152166,

E-mail: m.franzini@sssup.it; franzini@biomed.unipi.it

Received August 1, 2009; accepted December 23, 2009;

previously published online February 17, 2010
}

Keywords: $\gamma$-glutamyltransferase activity; $\gamma$-glutamyltransferase fractions; gel filtration chromatography; risk factors.

\section{Introduction}

Serum $\gamma$-glutamyltransferase (GGT) activity that is within its normal reference range was found to predict the risk of onset and evolution of atherosclerotic processes (1-3) and related diseases, such as hypertension $(4,5)$, type 2 diabetes (6-9), and metabolic syndrome (10). Evidence of the association between total serum GGT activity and coronary artery disease related events, infarction and stroke was achieved either in unselected populations (11-13) or in patients with known cardiovascular disease (14), thus confirming earlier epidemiological observations showing the predictive value of GGT for overall and cardiac mortality (15-17). GGT has been proposed as a marker of oxidative stress and exposure to xenobiotics $(18,19)$. Biochemical and pathology studies conducted in our laboratory showed that GGT activities within the reference range catalyze oxidative reactions in the presence of iron ions that lead to the production of free radicals and reactive oxygen species and low density lipoprotein (LDL) oxidation $(20,21)$. The potential role of GGT in promoting plaque formation and evolution has been confirmed by the identification of catalytically active GGT in coronary, cerebral and carotid plaques, colocalized with oxidized lipoproteins and CD68 + foam cells $(2,22)$. Plaque GGT has also been characterized. We recently showed, using a novel high performance gel filtration chromatography method, that a) circulating GGT is comprised of a combination of several distinct GGT-containing molecular complexes [b-GGT, m-GGT, s-GGT, and f-GGT, with molecular weight $(\mathrm{MW})>2000,940,140$, and $70 \mathrm{kDa}$, respectively], with s-GGT and f-GGT representing the greatest fractions in the plasma of healthy subjects $(23,24)$, b) GGT found in plaques has a MW that corresponds to b-GGT (25).

This study was performed to identify the determinants of the plasma GGT fraction pattern, in particular b-GGT in healthy subjects, and their association with established cardiovascular risk factors.

\section{Materials and methods}

\section{Subjects}

Fasting blood samples were obtained between 08:00 and 09:00 $\mathrm{h}$ from 200 healthy individuals [blood donors, 100 males, mean \pm standard deviation (SD), age $44 \pm 10$ years (range, 21-64 years),

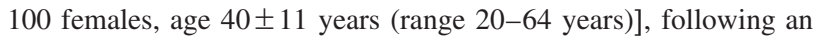
overnight fasting. The presence of acute or chronic diseases and of 


\section{Author's Copy}

714 Franzini et al.: $\gamma$-Glutamyltransferase fractions

metabolic syndrome was excluded by history, clinical examination and comprehensive laboratory testing. Metabolic syndrome was assessed using World Health Organization (WHO) $(26,27)$ criteria. These criteria used for this diagnosis were: fasting glucose $>6.11 \mathrm{mmol} / \mathrm{L}$ plus any two of the following: antihypertensive medication and/or high blood pressure [diastolic arterial pressure (DAP) $\geq 90 \mathrm{~mm} \mathrm{Hg}$, systolic arterial pressure (SAP) $\geq 140 \mathrm{~mm}$ $\mathrm{Hg}$ ], plasma triglycerides (TG) $\geq 1.69 \mathrm{mmol} / \mathrm{L}$, high density lipoprotein cholesterol (HDL-C) $<0.91 \mathrm{mmol} / \mathrm{L}$ in men or $<1.01 \mathrm{mmol} / \mathrm{L}$ in women, body mass index $(\mathrm{BMI})>30 \mathrm{~kg} / \mathrm{m}^{2}$. Subjects taking pharmacological treatment for whatever reasons or with alcohol consumption $>45 \mathrm{~g}$ /day for men and $>30 \mathrm{~g} /$ day for women were excluded from the study ( $\mathrm{n}=37$ males, 33 females); 59 males and 96 females were alcohol abstainers, 41 males with daily alcohol consumption $<45 \mathrm{~g}$ and four females with daily alcohol consumption $<30$ g. Of the 200 subjects, nine males (BMI range: 30.1-35.5) and nine females (BMI range: 30.1-35.4) were considered obese, and three males were pre-diabetic. According to WHO recommendations, we set the limit of hazardous alcohol consumption to $<45 \mathrm{~g} /$ day for men and $<30 \mathrm{~g} /$ day for women (28), However, $40 \mathrm{~g} /$ day consumption was identified in three males only, and $25 \mathrm{~g}$ /day consumption in one female, out of 200 control subjects. Total and fractional GGT activities in this population had been characterized previously (24). The study was approved by the Institutional Ethics Committee and all subjects gave informed consent.

\section{Laboratory analyses}

Analyses of all blood samples were performed simultaneously using standard clinical laboratory procedures with automated analyzers (Beckman Synchron CX 9-PRO analyzer, Beckman Coulter Italia,
Milan, Italy; Abbott Cell-Dyn Saphire for blood cell count, Abbott Italia, Latina, Italy). LDL cholesterol (LDL-C) and creatinine clearance were calculated using the Friedewald and the Cockcroft-Gault formula, respectively. High sensitivity C-reactive protein (CRP) was measured using the hsC Immunolite kit (Siemens Healthcare Diagnostics, Milan, Italy).

Analysis of total and fractional GGT was performed using plasma-EDTA (ethylenediaminetetraacetic acid) samples as described previously $(23,24)$ using an FPLC (fast protein liquid chromatography) system (AKTA purifier, GE Healthcare Europe, Milan, Italy) equipped with a gel filtration column (Superose 6 HR 10/300 GL, GE Healthcare Europe) and fluorescence detector (Jasco FP2020, Jasco Europe, Lecco, Italy). GGT activity was measured using $\gamma$-glutamyl-7-amido-4-methylcoumarin (gGluAMC, Nova Chimica, Milan, Italy) as substrate and glycylglycine as acceptor of the transpeptidation reaction. A $4.5 \mathrm{mmol} / \mathrm{L}$ stock solution of gGluAMC was prepared in ethanol $30 \% \mathrm{w} / \mathrm{w}$ containing $0.005 \mathrm{~N} \mathrm{NaOH}$ and stored at $-20^{\circ} \mathrm{C}$. This solution was diluted 25 -fold into $0.25 \mathrm{M}$ Tris- $\mathrm{HCl}$ buffer $\mathrm{pH} 8.5\left(25^{\circ} \mathrm{C}\right)$ daily.

\section{Statistics}

Biological parameters and values of GGT fractions between healthy males and females were compared using Student's t-test. Genderspecific analysis has been performed previously due to the wellknown difference in total GGT reference values and the potential difference to the association with recognized confounders in healthy subjects. To perform parametric statistical analysis, total, b-, m- and s-GGT, triglyceride and CRP values were ln-transformed to reduce skewness of the distribution. f-GGT fraction data were not ln-transformed because they were normally distributed (24).

Table 1 Characteristics of the study population.

\begin{tabular}{|c|c|c|c|}
\hline & $\begin{array}{l}\text { Males } \\
(n=100)\end{array}$ & $\begin{array}{l}\text { Females } \\
(n=100)\end{array}$ & p-Value \\
\hline Age, years & $44.0(9.5)$ & 39.9 (11.3) & $<0.01$ \\
\hline BMI, $\mathrm{kg} / \mathrm{m}^{2}$ & $25.8(2.8)$ & $23.6(4.0)$ & $<0.0001$ \\
\hline DAP, $\mathrm{mm} \mathrm{Hg}$ & $79.1(6.1)$ & $74.4(7.2)$ & $<0.0001$ \\
\hline $\mathrm{SAP}, \mathrm{mm} \mathrm{Hg}$ & $122.1(8.8)$ & $117.7(9.6)$ & $<0.001$ \\
\hline Blood glucose, $\mathrm{mmol} / \mathrm{L}$ & $5.39(0.57)$ & $5.00(0.57)$ & $<0.001$ \\
\hline Creatinine clearance, $\mathrm{mL} / \mathrm{s}$ & $1.88(0.44)$ & $1.72(0.43)$ & $<0.05$ \\
\hline Uric acid, $\mathrm{mmol} / \mathrm{L}$ & $0.32(0.07)$ & $0.24(0.06)$ & $<0.01$ \\
\hline Total cholesterol, $\mathrm{mmol} / \mathrm{L}$ & $4.94(1.00)$ & $4.81(1.04)$ & n.s. \\
\hline HDL-C, mmol/L & $1.25(0.34)$ & $1.52(0.39)$ & $<0.001$ \\
\hline LDL-C, $\mathrm{mmol} / \mathrm{L}$ & $3.23(0.84)$ & $2.95(0.88)$ & n.s. \\
\hline $\mathrm{TG}, \mathrm{mmol} / \mathrm{L}$ & $1.16(0.99)$ & $0.84(0.46)$ & $<0.001^{\mathrm{a}}$ \\
\hline AST (GO), U/L & $20.5(5.2)$ & $15.5(4.5)$ & $<0.0001$ \\
\hline ALT (GP), U/L & $24.3(9.8)$ & $13.6(6.0)$ & $<0.0001$ \\
\hline Total GGT, U/L & $30.6(17.5)$ & $16.1(6.9)$ & $<0.001^{\mathrm{a}}$ \\
\hline b-GGT, U/L & $3.6(3.3)$ & $1.6(1.6)$ & $<0.0001^{\mathrm{a}}$ \\
\hline m-GGT, U/L & $1.3(1.2)$ & $0.5(0.3)$ & $<0.0001^{\mathrm{a}}$ \\
\hline s-GGT, U/L & $12.4(11.4)$ & $5.0(3.9)$ & $<0.0001^{\mathrm{a}}$ \\
\hline f-GGT, U/L & $13.3(3.7)$ & $9.0(2.4)$ & $<0.0001$ \\
\hline Total bilirubin, $\mu \mathrm{mol} / \mathrm{L}$ & $14.67(6.70)$ & $11.92(3.30)$ & $<0.001$ \\
\hline Hemoglobin, g/L & $156.81(9.74)$ & $137.45(8.69)$ & $<0.0001$ \\
\hline $\mathrm{CRP}, \mathrm{mg} / \mathrm{L}$ & $1.53(2.65)$ & $1.90(2.89)$ & n.s. ${ }^{a}$ \\
\hline Leukocytes, $\times 10^{9} \mathrm{~L}$ & $6.6(1.7)$ & $6.5(1.5)$ & n.s. \\
\hline
\end{tabular}

Data are mean (SD). 'Student's t-test performed on ln-transformed data. BMI, body mass index; DAP, diastolic artery pressure; SAP, systolic artery pressure; HDL-C, high density lipoprotein cholesterol; LDL-C, low density lipoprotein cholesterol; TG, triglycerides; AST, aspartate aminotransferase; ALT, alanine aminotransferase; GGT, $\gamma$-glutamyltransferse; CRP, C-reactive protein; n.s., not significant. Creatinine clearance was estimated by the Cockroft-Gault formula; LDL-C was estimated by the Friedewald formula. 
Univariate linear correlations between biological variables, such as gender, age, BMI, estimated creatinine clearance, arterial pressure (AP), uric acid, plasma glucose, HDL-C and LDL-C, TG, aspartate aminotransferase (AST), alanine aminotransferase (ALT), CRP, and fractional GGT activity were presented as the Pearson correlation coefficient, and linear regression analyses were performed to assess associations. The association between alcohol consumption (considered as a dichotomous variable, that is individual consumption greater than or less than $12 \mathrm{~g} /$ day) and total or fractional GGT was analyzed using Student's t-test in males. Significant ethanol use was observed in three females only.

Multiple linear regression with total and fractional GGT activities as dependent variables was performed to quantify the independent effect of univariate significant factors. Results were presented as a $\beta$-standardized coefficient. $p<0.05$ was considered significant. Statistical analysis was performed with SPSS 13 analysis software (Copyright SPSS Inc.).

\section{Results}

\section{Characteristics of subjects}

Males showed significantly higher values for BMI, SAP, DAP, blood glucose, creatinine clearance, uric acid, serum TG, AST, ALT, total and fractional GGT, bilirubin, hemoglobin and lower HDL-C compared with females (Table 1). Total cholesterol and LDL-C, CRP levels and leukocytes count were not different between the two populations.

\section{Determinants of GGT fraction activity according to gender}

All GGT fractions were associated with ALT in both genders (Table 2). All GGT fractions were associated with AST in males, whereas in females AST was associated with m-GGT and f-GGT only (Table 2). Total and fractional GGT was not significantly associated with creatinine clearance in healthy subjects (not shown).

In healthy males, all GGT fractions were associated with uric acid, TG, AST, CRP and leukocyte count. In addition, b-GGT activity was associated with BMI, DAP, LDL-C and hemoglobin; m-GGT was associated with LDL-C and negatively associated with HDL-C; s-GGT was associated with hemoglobin; f-GGT was associated with BMI, DAP, LDL-C and hemoglobin, while being negatively associated with HDL-C. Moderate alcohol consumption did not significantly influence total and fractional GGT in male subjects (Supplemental data Table 1).

In females, all GGT fractions were significantly associated with SAP, DAP and hemoglobin. In addition, b-GGT activity was associated with age, BMI, glucose, uric acid, LDL-C, TG, CRP and leukocyte count; m-GGT was associated with BMI, glucose, uric acid, LDL-C, TG, AST, CRP and leukocyte count; s-GGT was associated with TG, CRP and leukocyte count; f-GGT was associated with age, glucose, LDL-C, AST and bilirubin (Table 2). 
Table 3 Multivariate linear regression analysis: independent predictors of GGT fraction activity in healthy subjects.

\begin{tabular}{cllllll}
\hline & Gender & DAP & LDL-C & TG & ALT & CRP \\
\hline Total GGT & $0.249^{\mathrm{a}}$ & n.s. & n.s. & n.s. & $0.432^{\mathrm{a}}$ & $0.132^{\mathrm{b}}$ \\
b-GGT & n.s. & $0.153^{\mathrm{b}}$ & n.s. & $0.188^{\mathrm{c}}$ & $0.379^{\mathrm{a}}$ & $0.148^{\mathrm{b}}$ \\
m-GGT & n.s. & n.s. & $0.283^{\mathrm{a}}$ & $0.168^{\mathrm{c}}$ & $0.394^{\mathrm{a}}$ & $0.142^{\mathrm{b}}$ \\
s-GGT & $0.255^{\mathrm{a}}$ & n.s. & n.s. & $0.141^{\mathrm{b}}$ & $0.448^{\mathrm{a}}$ & $0.197^{\mathrm{c}}$ \\
f-GGT & $0.273^{\mathrm{a}}$ & n.s. & $0.173^{\mathrm{c}}$ & n.s. & $0.290^{\mathrm{a}}$ & n.s. \\
\hline
\end{tabular}

Data reported are $\beta$ standardized coefficients. ${ }^{\mathrm{a}} \mathrm{p} \leq 0.001,{ }^{\mathrm{b}} \mathrm{p}<0.05,{ }^{\mathrm{c}} \mathrm{p} \leq 0.01$. DAP, diastolic artery pressure; LDL-C, low density lipoprotein cholesterol; TG, triglycerides; ALT, alanine aminotransferase; CRP, C-reactive protein; GGT, $\gamma$-glutamyltransferase; n.s., not significant.

\section{Independent predictors of GGT fraction activity in healthy subjects}

Total GGT was independently associated with gender, CRP and ALT. The latter was independently associated with all GGT fractions. TG and CRP were independently associated with b-, m- and s-GGT; in addition DAP was associated only with b-GGT, LDL-C with m-GGT and gender with s-GGT. The latter two variables were positively associated also with f-GGT (Table 3). No association was seen with respect to age, BMI, glucose and HDL-C (data not shown).

\section{Discussion}

This work is the first to show that in healthy individuals, GGT fractions show different associations with established cardiovascular risk factors, namely those that are markers of inflammation (CRP) and lipid metabolism.

In particular, while gender was found to correlate independently only with the two low MW forms (s-GGT and fGGT), and ALT did not show specific association with any GGT fraction, well-known cardiovascular risk factors, such as DAP, CRP, TG and LDL-C correlated primarily with the higher MW fractions (b-GGT, the form identified within atherosclerotic plaques, and m-GGT).

GGT activities within the reference range display pro-oxidant properties (21), being able to catalyze the oxidation of LDL (20), and catalytically active GGT was found within the plaque where it is co-localized with oxidized LDL and CD68 + foam cells (22). Furthermore, GGT activities within the reference range predicts the risk of onset and evolution of atherosclerotic processes (1-3).

A key point to explaining the link between GGT and cardiovascular events is the relationship between GGT and stability of the atherosclerotic plaque and to establish how circulating GGT contributes to GGT activity. Recent studies show that GGT activity present in the plaque is proportional to that found in blood, displaying the same MW of plasma b-GGT. This suggests that only b-GGT can enter the arterial wall and accumulate within the plaque (25). These results are in agreement with the finding that increases in total GGT activity, but within the reference values, is associated primarily with an increase in s-GGT, b-GGT and m-GGT (24). The main advantage of the FPLC method for analysis of plasma GGT activity is the ability to directly quantify and study the clinical significance of each fraction, independently from the behavior of the other fractions.

Gender, the only well-known physiological determinant of total GGT (1), was found to be an independent determinant of the lower MW s-GGT and f-GGT fractions only, whereas markers with known predictive value for cardiovascular events (such as AP, TG, CRP) showed a common association with the potentially atherogenic b-GGT, but not with f-GGT.

Despite that total GGT was recognized as a predictor of diabetes and the metabolic syndrome (10), GGT fractions did not show an independent association with BMI and serum glucose. This suggests that the predictive value of GGT for these conditions is not a mere consequence of glucose concentrations or metabolic overload on the liver. Instead, this is due to the complex interplay between inflammation, abnormal lipid metabolism and hepatocellular damage demonstrated by high MW GGT fractions determinants (CRP, ALT, TG, LDL-C). Notably, none of our healthy individuals that we recruited fulfilled criteria for the metabolic syndrome, and alcohol consumption did not influence GGT fractional values. Measurement of waist circumference, which has not been obtained in this subset, is well correlated with intra-abdominal visceral adipose tissue accumulation, and could add relevant information.

These studies were performed in a highly selected group of apparently healthy individuals. It is questionable whether this information applies to the understanding of the potential association of subfractions of GGT with cardiovascular disease. For this purpose, follow-up of these findings in cohort studies with validated end points (cardiovascular morbidity and mortality) should be performed. These findings might help in the understanding of the pathogenetic association between GGT activity and cardiovascular disease. In addition, the ability to assess GGT fractions will help overcome the limit represented by low specificity of serum GGT determination either for diagnostic or prognostic purposes.

Supplementary data associated with this article can be found in the online version at: http://www.reference-global.com/doi/suppl/ 10.1515/CCLM.2009.125.

\section{Conflict of interest statement}

Authors' conflict of interest disclosure: The authors stated that there are no conflicts of interest regarding the publication of this article. Research funding played no role in the study design; in the 
collection, analysis, and interpretation of data; in the writing of the report; or in the decision to submit the report for publication.

Research funding: This work was supported by Institutional funding (G. Monasterio Foundation CNR-Regione Toscana, Scuola Sant' Anna, and University of Pisa, Italy).

Employment or leadership: None declared.

Honorarium: None declared.

\section{References}

1. Whitfield JB. Gamma glutamyl transferase. Crit Rev Clin Lab Sci 2001;38:263-355.

2. Paolicchi A, Emdin M, Ghliozeni E, Ciancia E, Passino C, Popoff G, et al. Human atherosclerotic plaques contain gammaglutamyl transpeptidase enzyme activity [Images in cardiovascular medicine]. Circulation 2004;109:1440.

3. Emdin M, Pompella A, Paolicchi A. Gamma-glutamyltransferase, atherosclerosis, and cardiovascular disease: triggering oxidative stress within the plaque [editorial]. Circulation 2005; 112:2078-80.

4. Lee DH, Jacobs DR Jr, Gross M, Kiefe CI, Roseman J, Lewis $\mathrm{CE}$, et al. Gamma-glutamyltransferase is a predictor of incident diabetes and hypertension: the Coronary Artery Risk Development in Young Adults (CARDIA) study. Clin Chem 2003;49: 1358-66.

5. Lee DH, Jacobs DR Jr, Gross M, Steffes M. Serum gammaglutamyltransferase was differently associated with microalbuminuria by status of hypertension or diabetes: the Coronary Artery Risk Development in Young Adults (CARDIA) study. Clin Chem 2005;51:1185-91.

6. Lee DH, Silventoinen K, Jacobs DR Jr, Jousilahti P, Tuomileto J. Gamma-glutamyltransferase, obesity, and the risk of type 2 diabetes: observational cohort study among 20,158 middle-aged men and women. J Clin Endocr Metab 2004;89:5410-4.

7. Lim JS, Lee DH, Park JY, Jin SH, Jacobs DR Jr. A strong interaction between serum gamma-glutamyltransferase and obesity on the risk of prevalent type 2 diabetes: results from the Third National Health and Nutrition Examination Survey. Clin Chem 2007;53:1092-8.

8. Targher G, Zoppini G, Lippi G, Guidi GC, Muggeo M. Effect of serum gamma-glutamyltransferase and obesity on the risk of dyslipidemia and poor glycemic control in type 2 diabetic patients: cross-sectional findings from the Verona diabetes study. Clin Chem 2007;53:1867-9.

9. Lippi G, Targher G, Guidi GC. Relationship between gammaglutamyltransferase, fasting plasma glucose, and triglycerides in the general population. Clin Chem 2007;53:1866-7.

10. Lee DS, Evans JC, Robins SJ, Wilson PW, Albano I, Fox CS, et al. Gamma glutamyl transferase and metabolic syndrome, cardiovascular disease, and mortality risk: the Framingham Heart study. Arterioscler Thromb Vasc Biol 2007;27:127-33.

11. Ruttmann E, Brant LJ, Concin H, Diem G, Rapp K, Ulmer H. Gamma-glutamyltransferase as a risk factor for cardiovascular disease mortality. An investigation in a cohort of 163,944 Austrian adults. Circulation 2005;112:2130-7.

12. Lee DH, Silventoinen K, Hu G, Jacobs DR Jr, Jousilahti P, Sundvall J, et al. Serum gamma-glutamyltransferase predicts non-fatal myocardial infarction and fatal coronary heart disease among 28,838 middle-aged men and women. Eur Heart J 2006;27:2170-6.

13. Meisinger C, Doring A, Schneider A, Lowel H, KORA Study Group. Serum gamma-glutamyltransferase is a predictor of inci- dent coronary events in apparently healthy men from the general population. Atherosclerosis 2006;189:297-302.

14. Emdin M, Passino C, Michelassi C, Titta F, L'Abbate A, Donato $\mathrm{L}$, et al. Prognostic value of serum gamma-glutamyl transferase activity after myocardial infarction. Eur Heart J 2001; 22:1802-7.

15. Conigrave KM, Saunders JB, Reznik RB, Whitfield JB. Prediction of alcohol-related harm by laboratory test results. Clin Chem 1993;39:2266-70.

16. Wannamethee G, Ebrahim S, Shaper AG. Gamma-glutamyltransferase: determinants and association with mortality from ischaemic heart disease and all causes. Am J Epidemiol 1995; 142:699-708.

17. Brenner H, Rothenbacher D, Arndt V, Schuberth S, Fraisse E, Fliedner TM. Distribution, determinants, and prognostic value of gamma-glutamyltranspeptidase for all-cause mortality in a cohort of construction workers from south Germany. Prev Med 1997;26:305-10.

18. Lee DH, Blomhoff R, Jacobs DR Jr. Is serum gamma glutamyltransferase a marker of oxidative stress? Free Radic Res 2004;38:535-9.

19. Lee DH, Jacobs DR Jr. Association between serum concentrations of persistent organic pollutants and gamma glutamyltransferase: results from the National Health and Examination Survey 1999-2002. Clin Chem 2006;52:1825-7.

20. Paolicchi A, Minotti G, Tonarelli P, Tongiani R, De Cesare D, Mezzetti A, et al. Gamma-glutamyl transpeptidase-dependent iron reduction and LDL oxidation - a potential mechanism in atherosclerosis. J Investig Med 1999;47:151-60.

21. Dominici S, Paolicchi A, Lorenzini E, Maellaro E, Comporti M, Pieri L, et al. Gamma-glutamyltransferase-dependent prooxidant reactions: a factor in multiple processes. Biofactors 2003;17:187-98.

22. Emdin M, Passino C, Donato L, Paolicchi A, Pompella A. Serum gamma-glutamyltransferase as a risk factor of ischemic stroke might be independent of alcohol consumption. Stroke 2002;33:1163-4.

23. Franzini M, Bramanti E, Ottaviano V, Ghiri E, Scatena F, Barsacchi R, et al. A high performance gel filtration chromatography method for gamma-glutamyltransferase fraction analysis. Anal Biochem 2008;374:1-6.

24. Franzini M, Ottaviano V, Fierabracci V, Bramanti E, Zyw L, Barsacchi R, et al. Fractions of plasma gamma-glutamyltransferase in healthy individuals: reference values. Clin Chim Acta 2008;395:188-9.

25. Franzini M, Corti A, Martinelli B, Del Corso A, Emdin M, Parenti GF, et al. Gamma-glutamyltransferase activity in human atherosclerotic plaques - biochemical similarities with the circulating enzyme. Atherosclerosis 2009;202:119-27.

26. Alberti KG, Zimmet PZ. Definition, diagnosis and classification of diabetes mellitus and its complications. Part 1: diagnosis and classification of diabetes mellitus provisional report of a WHO consultation. Diabet Med 1998;15:539-53.

27. World Health Organization. Definition, diagnosis and classification of diabetes mellitus and its complications: report of a WHO Consultation. Part 1: diagnosis and classification of diabetes mellitus. Geneva, Switzerland: World Health Organization; 1999. Available at: http://whqlibdoc.who.int/hq/1999/ WHO_NCD_NCS_99.2.pdf Accessed April 2009.

28. Schellenberg F, Schwan R, Mennetrey L, Loiseaux MN, Pagès JC, Reynaud M. Dose-effect relation between daily ethanol intake in the range 0-70 grams and \%CDT value: validation of a cut-off value. Alcohol Alcohol 2005;40:531-4. 


\section{Supplemental Data}

Supplementary Table 1 Total and fractional GGT activity in healthy males, either abstainers, or with moderate alcohol use [mean (SD): $18.2(11.7) \mathrm{g} / \mathrm{day}]$.

\begin{tabular}{|c|c|c|c|c|c|}
\hline & \multicolumn{2}{|c|}{ Abstainers $(\mathrm{n}=59)$} & \multicolumn{2}{|c|}{ Alcohol users $(n=41)$} & \multirow[t]{2}{*}{ p-Value } \\
\hline & Median & Range & Median & Range & \\
\hline Total GGT & 24.7 & $12.1-60.6$ & 26.1 & $14.8-52.6$ & n.s. \\
\hline b-GGT ${ }^{a}$ & 2.3 & $0.6-11.8$ & 2.5 & $1.2-8.7$ & n.s. \\
\hline $\mathrm{m}-\mathrm{GGT}^{\mathrm{a}}$ & 0.9 & $0.3-2.8$ & 1.3 & $0.2-3.3$ & n.s. \\
\hline $\mathrm{s}-\mathrm{GGT}^{\mathrm{a}}$ & 7.3 & $2.4-33.9$ & 10.3 & $3.5-25.4$ & n.s. \\
\hline f-GGT & 12.4 & $7.8-19.6$ & 13.7 & $9.2-19.6$ & n.s. \\
\hline
\end{tabular}

Data are expressed as U/L. ${ }^{\mathrm{a}} \mathrm{t}$-Test performed on $\ln$-transformed data. n.s., not significant. 